\title{
Wogonin induces Beclin-1/PI3K and reactive oxygen species-mediated autophagy in human pancreatic cancer cells
}

\author{
SHAO-JUN LI* ${ }^{*}$ SHI-JIE SUN, JIE GAO and FU-BO SUN* \\ Department of Hepatobiliary and Pancreatic Surgery, The Affiliated Yantai Yuhuangding Hospital of Qingdao University, \\ Yantai, Shandong 264000, P.R. China
}

Received July 2, 2015; Accepted August 26, 2016

DOI: $10.3892 / \mathrm{ol} .2016 .5367$

\begin{abstract}
Wogonin is considered to be an inhibitor of myeloid cell leukemia 1 and B-cell lymphoma 2, and a potential antitumor drug due to its ability to induce apoptosis in certain cancer cells; however, few previous studies have reported on wogonin-induced autophagy. The aim of the present study was to investigate the influence of wogonin on autophagy in human pancreatic cancer cells (HPCCs), elucidate its mechanism, and identify strategies to increase its effectiveness as an anti-cancer treatment. HPCCs were treated with wogonin and autophagy was detected in the cells. The mechanism of wogonin-related autophagy was investigated, and the antioxidant $\mathrm{N}$-acetyl-L-cysteine (NAC) was used to assess the role of reactive oxygen species (ROS) in wogonin-related autophagy. The results demonstrated that wogonin may induce autophagy by activating the Beclin-1/phosphatidylinositol-3-kinase and ROS pathways in HPCCs, and may enhance ROS generation, followed by the activation of the AKT/ULK1/4E-BP1/CYLD pathway and inhibition of the mammalian target of rapamycin signaling pathway. The incubation of HPCCs with wogonin and the antioxidant NAC, revealed that the effects of wogonin-enhanced ROS generation on autophagy-related molecules were inhibited, contributing to the inhibition of autophagy and increasing the cell death ratio through apoptosis activation in HPCCs. These studies suggest that autophagy activation, via the ROS pathway, by the antitumor drug wogonin in HPCCs may partially reduce the antitumor effects of the drug, and that the
\end{abstract}

Correspondence to: Dr Fu-Bo Sun, Department of Hepatobiliary and Pancreatic Surgery, The Affiliated Yantai Yuhuangding Hospital of Qingdao University, 20 Yuhuangding Road, Yantai, Shandong 264000, P.R. China

E-mail: fubosun_1@163.com

*Contributed equally

Abbreviations: ROS, reactive oxygen species; NAC, $\mathrm{N}$-acetyl-L-cysteine; HPCCs, human pancreatic cancer cells

Key words: wogonin, reactive oxygen species, autophagy, human pancreatic cancer cells antioxidant NAC may enhance the antitumor effectiveness of wogonin via the inhibition of ROS-enhanced autophagy and the subsequent promotion of apoptosis. Therefore, the present research suggests that wogonin combined with NAC may be a novel combination therapy for clinical pancreatic cancer therapy trials.

\section{Introduction}

Pancreatic cancer has a mortality rate of $>95 \%$, which has not improved for 3 decades (1). The current treatment of pancreatic carcinoma consists of a combination of chemotherapy (including gemcitabine), radiotherapy and surgery, although the treatment may vary (2). The majority of patients with pancreatic cancer are diagnosed at a late and inoperable stage and the 5-year survival rate is poor (3). In investigating novel therapeutic and preventative strategies, a number of studies have focused on developing novel chemotherapeutic agents. For example, Lewis et al (4) have demonstrated that the sphingosine kinase-2 inhibitor ABC294640 acts as a chemotherapeutic agent for the treatment of pancreatic cancer through suppression of c-Myc and RRM2 expression. In addition, Mandhare et al (5) reviewed the effect of Azaepothilone B on chemotherapeutic medication for pancreatic cancer. Pourmorteza et al (6) demonstrated that evofosfamide targets tumor hypoxia, and may be a potential agent against pancreatic cancer. Although numerous novel chemotherapeutic agents have been identified, their antitumor mechanisms, and how these may be manipulated to promote antitumor efficacy, are not clear at present.

Wogonin (5,7-dihydroxy-8-methoxyflavone) is a potent inhibitor of myeloid cell leukemia 1 (Mcl-1) and B-cell lymphoma 2 (Bcl-2), which are anti-apoptotic proteins that are expressed in various tumors, including pancreatic cancer $(7,8)$. Previous studies $(9,10)$ have demonstrated the antitumor potential of wogonin, particularly its ability to induce apoptosis and its high maximum-tolerated dose. Wogonin has also been observed to be a potential anti-pancreatic cancer drug through its ability to inhibit the Mcl-1 and nuclear factor- $\kappa \mathrm{B}$ (NF-кB) pathways $(11,12)$.

Autophagy has become a novel target for the treatment of certain types of cancer, and may prevent cell death (13). Few studies have previously reported wogonin-induced autophagy $(14,15)$. In present study, wogonin was added into 
the medium of HPCCs, and the influence of wogonin on autophagy and its mechanism were investigated. Furthermore, the antioxidant $\mathrm{N}$-acetyl-L-cysteine (NAC) was used as a chemosensitizer of wogonin to detect whether it can enhance cancer cell death, and its mechanism was investigated.

\section{Materials and methods}

Cell culture. Panc-1 and Colo-357 HPCCs were obtained from the American Type Culture Collection (Manassas, VA, USA). All cells were cultured in Dulbecco's modified Eagle's medium (DMEM) supplemented with $10 \%$ fetal bovine serum (FBS), $2 \mathrm{mM}$ L-glutamine, $100 \mathrm{U} / \mathrm{ml}$ penicillin and $100 \mathrm{mg} / \mathrm{ml}$ streptomycin at $37^{\circ} \mathrm{C}$ in an atmosphere containing $5 \% \mathrm{CO}_{2}$. HPCCs were treated with $40 \mu \mathrm{M}$ wogonin, $40 \mu \mathrm{M}$ wogonin combined with $40 \mu \mathrm{M}$ chloroquine (CQ), or $50 \mu \mathrm{M}$ rapamycin for $24 \mathrm{~h}$; in addition, HPCCs were pretreated with sterile water or $10 \mathrm{mM}$ NAC for $2 \mathrm{~h}$ and then treated with $0.1 \%$ DMSO or $40 \mu \mathrm{M}$ wogonin for $24 \mathrm{~h}$. All cell culture reagents were purchased from Gibco (Thermo Fisher Scientific, Inc., Waltham, MA, USA).

Western blotting. The cells $\left(5 \times 10^{6}\right)$ were lysed for $30 \mathrm{~min}$ in radioimmunoprecipitation assay lysis buffer $[50 \mathrm{mM}$ Tris ( $\mathrm{pH} 7.4$ ), $150 \mathrm{mM} \mathrm{NaCl}, 1 \%$ Triton $\mathrm{X}-100,1 \%$ sodium deoxycholate, $0.1 \%$ SDS; Beyotime Institute of Biotechnology, Haimen, China] on ice then centrifuged at $12,000 \times \mathrm{g}$ for $12 \mathrm{~min}$ at $4^{\circ} \mathrm{C}$. A bicinchoninic acid assay (Beyotime Institute of Biotechnology) was used to determine the protein concentration. The proteins were separated by $8-15 \%$ sodium dodecyl sulfate polyacrylamide gel electrophoresis, and blotted onto a polyvinylidene difluoride membrane (PVDF) following blocking with $5 \%$ nonfat dry milk in phosphate buffered saline (PBS) with $0.05 \%$ Tween 20 (PBST). The membranes were incubated with the primary antibodies (dilution, 1:1,000) for $2 \mathrm{~h}$ at $37^{\circ} \mathrm{C}$, then the PVDF membranes were washed three times for 10 min in PBST. Secondary antibodies (dilution, 1:10,000) were incubated for $1 \mathrm{~h}$ at $37^{\circ} \mathrm{C}$ and the PVDF membranes were then washed three times for $10 \mathrm{~min}$ in PBST. The antibodies used included rabbit polyclonal IgG primary antibodies against LC3 $\alpha$ (\#sc-134226), Beclin-1 (\#sc-11427), phosphatidylinositol 3-kinase (PI3K; \#sc-134766), total mammalian target of rapamycin (mTOR; \#sc-8319), phospho-mTOR (\#sc-101738), Unc-51-like autophagy-activating kinase 1 (ULK1; \#sc-33182) and cylindromatosis (CYLD; \#sc-28211), mouse monoclonal $\mathrm{IgG}_{1}$ primary antibodies against protein kinase B (AKT; \#sc-5298), eukaryotic initiation factor 4E-binding protein 1 (4E-BP1; \#sc-9977) and GAPDH (\#sc-365062), and anti-mouse IgG (\#sc-2380) and anti-rabbit IgG (\#sc-2385) secondary antibodies. All antibodies were purchased from Santa Cruz Biotechnology, Inc. (Dallas, TX, USA). Subsequently, the PVDF membranes were exposed to BeyoECL Star (\#P0018A; Beyotime Institute of Biotechnology) and ECL-sensitive films, and developed by OPTIMAX X-Ray film processor (Optimax 2010; Protec GmbH \& Co. KG, Oberstenfeld, Germany).

Acridine orange (AO) staining. The degradation of autophagosomes is mediated by lysosomes; autolysis lowers the $\mathrm{pH}$ value and allows AO dye to penetrate into the acidic organelles and exhibit a red fluorescence; therefore, the intensity of red fluorescence following AO staining can be used to indicate the levels of autolysosomes (16). Cells were treated with dimethyl sulfoxide (DMSO) or $40 \mu \mathrm{M}$ wogonin for $24 \mathrm{~h}$ in DMEM supplemented with $10 \% \mathrm{FBS}$ at $37^{\circ} \mathrm{C}$ in an atmosphere containing $5 \% \mathrm{CO}_{2}$, then washed three times with PBS, fixed with $4 \%$ paraformaldehyde for $15 \mathrm{~min}$, stained with $1 \mathrm{mg} / \mathrm{l} \mathrm{AO}$ dye solution for $10 \mathrm{~min}$ at $37^{\circ} \mathrm{C}$ and then imaged under a fluorescence microscope (Nikon TE2000; Nikon Corporation, Tokyo, Japan).

Green fluorescent protein (GFP)-conjugated microtubule-associated protein 1A/1B-light chain 3 (LC3) analysis. When autophagy is induced, cytosolic LC3 is cleaved by hydrolysis to a shorter peptide (LC3 II) that is located on the membrane of the autophagosome; therefore, the expression levels of LC3 II may be used to estimate the level of autophagy. In order to estimate the location and processing of LC3-II, assessment of LC3-GFP puncta is an effective method (16). Panc-1 and Colo-357 cells were incubated in 6-well plates with DMEM supplemented with $10 \% \mathrm{FBS}$ at $37^{\circ} \mathrm{C}$ in an atmosphere containing $5 \% \mathrm{CO}_{2}$. When they had reached $80 \%$ confluence, the cells were incubated with Opti-MEM medium (Thermo Fisher Scientific, Inc.), and transfected with a GFP-LC3 vector (GeneChem Co., Ltd., Shanghai, China) using Lipofectamine ${ }^{\circledR} 2000$ (Invitrogen; Thermo Fisher Scientific, Inc.) for $24 \mathrm{~h}$. Subsequently, the cells were treated with DMSO or $40 \mu \mathrm{M}$ wogonin for $24 \mathrm{~h}$, then fixed with $4 \%$ paraformaldehyde for $15 \mathrm{~min}$, and washed three times with PBS. The cells were imaged under a fluorescence microscope (Nikon TE2000) and LC3-GFP puncta-positive cells were counted ( $\geq 5$ puncta was considered positive).

Co-immunoprecipitation experiments. Panc-1 cells $\left(5 \times 10^{6}\right)$ were plated onto $15-\mathrm{cm}$ dishes and attached overnight. The cells were treated with DMSO or $40 \mu \mathrm{M}$ wogonin for $12 \mathrm{~h}$ at $37^{\circ} \mathrm{C}$ then lysed for $30 \mathrm{~min}$ in hypotonic lysis buffer (Beyotime Institute of Biotechnology) on ice and centrifuged (1,000 x $g$, $\left.4^{\circ} \mathrm{C}, 15 \mathrm{~min}\right)$ as a whole-cell lysate. Whole-cell lysates were precleared with protein A-agarose (50\% in PBS), and then incubated with antibodies against Beclin-1 or PI3K (\#sc-11427 and \#sc-134766; Santa Cruz Biotechnology, Inc.; dilution, 1:300) for $1 \mathrm{~h}$ at $4^{\circ} \mathrm{C}$. The immunoprecipitates were captured on a protein-A agarose gel and then detected by immnuoblotting; whole-cell lysates and immunoprecipitates were separated by $12 \%$ SDS-PAGE and transferred to PVDF membranes for detection, as described above (17).

Cell viability assay. Cell Counting kit-8 (CCK8) was used to assess cell viability. Cells $\left(1 \times 10^{4}\right)$ were seeded into a 96-well plate and incubated overnight in the previously described conditions. The cells were pretreated with sterile water or $10 \mathrm{mM}$ NAC for $2 \mathrm{~h}$ and then with $0.1 \%$ DMSO or $40 \mu \mathrm{M}$ wogonin for $24 \mathrm{~h}$. Following this, the medium was removed and the cells were washed three times with PBS. DMEM (90 $\mu \mathrm{l})$ and CCK8 $(10 \mu \mathrm{l})$ were subsequently added to each well and incubated for $1.5 \mathrm{~h}$ at $37^{\circ} \mathrm{C}$; a microplate reader was used to measure the optical density (OD) at $450 \mathrm{~nm}$.

ROS detection. The levels of intracellular ROS generation were detected through measuring the conversion of cell-permeable 
$\mathbf{A}$

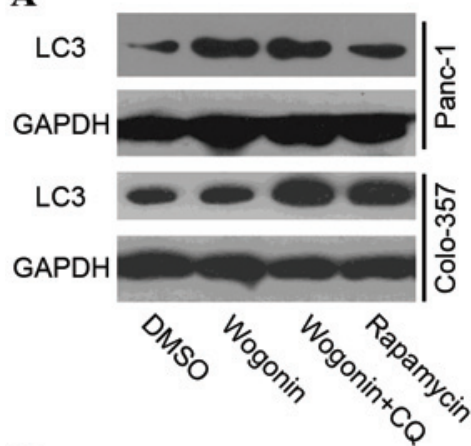

D

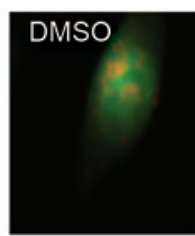

DMSO
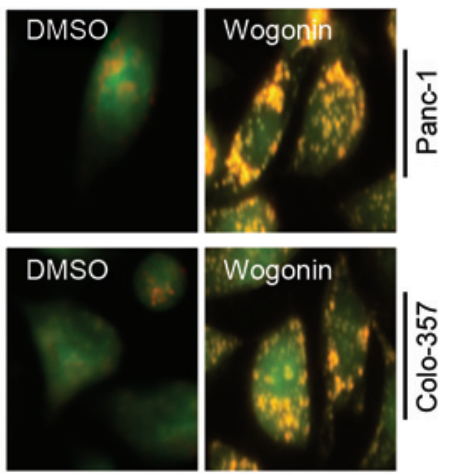

B

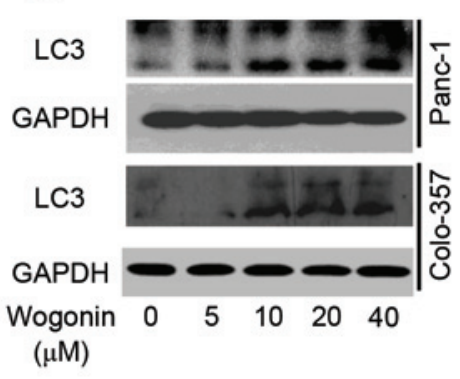

$\mathbf{E}$
C

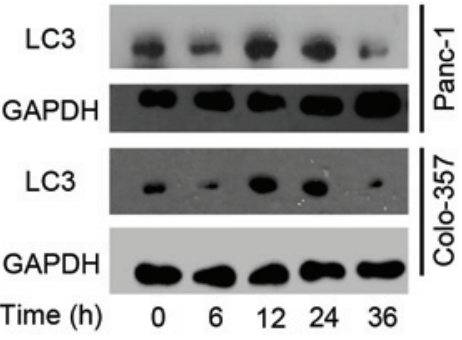

F

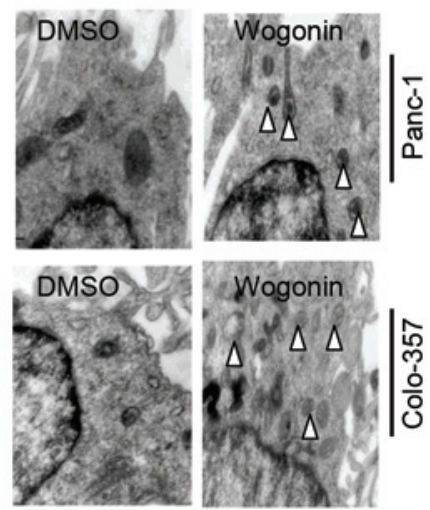

Figure 1. Wogonin induces autophagy in HPCC. (A) HPCCs were treated with $40 \mu \mathrm{M}$ wogonin, wogonin combined with $40 \mu \mathrm{M}$ CQ or $50 \mu \mathrm{M}$ rapamycin for $24 \mathrm{~h}$; LC3 expression was induced by wogonin, indicating its ability to induce autophagy. (B) HPCCs were treated with various doses of wogonin for 24 h, and wogonin-induced autophagy was found to be dose-dependent, with an optimal dose of $40 \mu \mathrm{M}$. (C) HPCCs were treated with $40 \mu \mathrm{M}$ wogonin and incubated for various times; wogonin-induced autophagy was time-dependent (optimal time, $24 \mathrm{~h}$ ). (D) HPCCs were treated with $40 \mu \mathrm{M}$ wogonin and processed for fluorescent microscopy for $24 \mathrm{~h}$; the level of autolysosomes markedly increased following treatment with wogonin. (E) Following GFP-LC3 transfection, cells were treated with $0.1 \%$ DMSO or $40 \mu \mathrm{M}$ wogonin for $24 \mathrm{~h}$, and the LC3-GFP puncta-positive cells were counted ( $\geq 5$ puncta was considered positive); the number of LC3-GFP puncta-positive cells was increased with wogonin treatment $\left({ }^{* * *} \mathrm{P}<0.005\right)$. Data are presented as the mean \pm standard deviation from triplicated experiments. (F) TEM was used to detect autophagic vacuoles (arrows), indicating that wogonin treatment induced autophagy. HPCC, human pancreatic cancer cell; CQ, chloroquine, LC3, microtubule-associated protein 1A/1B-light chain 3; GFP, green fluorescent protein; TEM, transmission electron microscopy; GAPDH, glyceraldehyde 3-phosphate dehydrogenase; DMSO, dimethyl sulfoxide.

2,7-dichlorofluorescein diacetate (DCFH-DA; Beyotime Institute of Biotechnology) to fluorescent dichlorofluorescein (DCF). Cells $\left(1 \times 10^{4}\right)$ were seeded into 96-well plates and incubated overnight in the standard conditions. The cells were pretreated with sterile water or $10 \mathrm{mM} \mathrm{NAC}$ for $2 \mathrm{~h}$ and then treated with $0.1 \%$ DMSO or $40 \mu \mathrm{M}$ wogonin for $24 \mathrm{~h}$. The medium was removed and the cells were washed three times with PBS prior to incubation with DCFH-DA at $37^{\circ} \mathrm{C}$ for $20 \mathrm{~min}$. A fluorescence microplate reader, with $488 \mathrm{~nm}$ excitation wavelength and $525 \mathrm{~nm}$ emission wavelength, was used to determine the levels of ROS in the cells.

Detection of glutathione (GSH) and superoxide anions $\left(\mathrm{O}_{2}^{-}{ }^{-}\right)$. The intracellular levels of GSH were measured by GSH Assay kit (Beyotime Institute of Biotechnology) according to the manufacturer's protocol. The OD value of GSH was detected with a fluorescence microplate reader (Tecan, Männedorf, Switzerland) at $420 \mathrm{~nm}$. In addition, the intracellular levels of $\mathrm{O}_{2}^{-}$were detected by Superoxide Assay kit (Beyotime Institute of Biotechnology), and the fluorescence value of $\mathrm{O}_{2}$ was measured at a wavelength of $550 \mathrm{~nm}$.

Trypan blue assay. Following pretreatment with sterile water or $10 \mathrm{mM} \mathrm{NAC}$ for $2 \mathrm{~h}$ and treatment with $0.1 \%$ DMSO or
$40 \mu \mathrm{M}$ wogonin for $24 \mathrm{~h}$, the cells were suspended in $0.25 \%$ Trypsin-EDTA (Thermo Fisher Scientific, Inc.), and 0.4\% (w/v) trypan blue solution was added to each $2-\mu l$ suspension; the ratio of the cell suspension to the trypan blue solution was 9:1. The cells were counted under a light microscope. As the dead cells fail to exclude the dye, the cell death rate was calculated using the following equation: Total cell death rate $=($ no. dyed cells $/$ total no. cells) $\times 100 \%$.

Transmission electron microscopy (TEM). TEM is the gold standard for the assessment of autophagosomes (16). Samples were prepared using a previously described method (18). Briefly, the treated cells were washed three times with PBS times, suspended in $0.25 \%$ Trypsin-EDTA, rinsed three times in the distilled water and dehydrated in the different concentrations of ethanol $(50,70,80,90,95$ and 100\%; 8 min each). Subsequently, the cells were placed into propylene oxide for $10 \mathrm{~min}$, and then into Embed 812 Resin medium and propylene oxide mixture (1:1) overnight at room temperature. Samples were then cured in BEEM capsules overnight at $60^{\circ} \mathrm{C}$ and the sections were cut at $50 \mathrm{~nm}$ by use of an ultramicrotome (EM UC7/FC7; Leica Biosystems, Nussloch, Germany). The cut sections were stained with lead citrate and $2 \%(\mathrm{w} / \mathrm{v})$ uranyl acetate for TEM detection. TEM was 
A

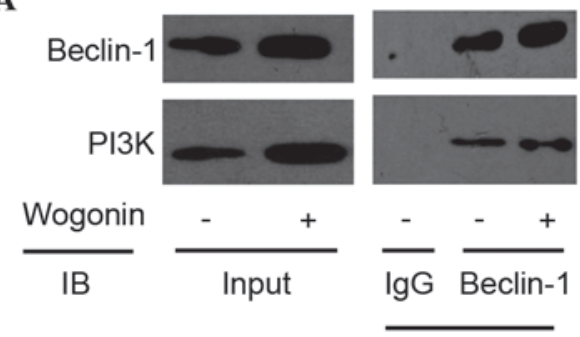

IP

C

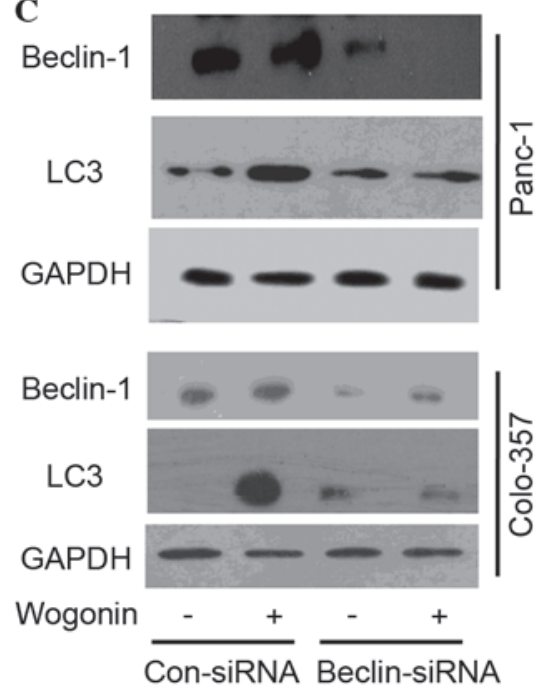

B

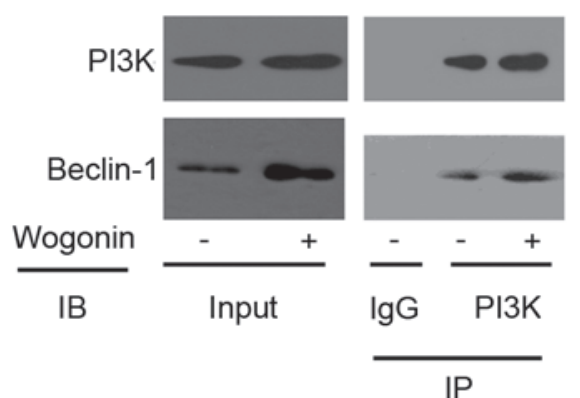

D
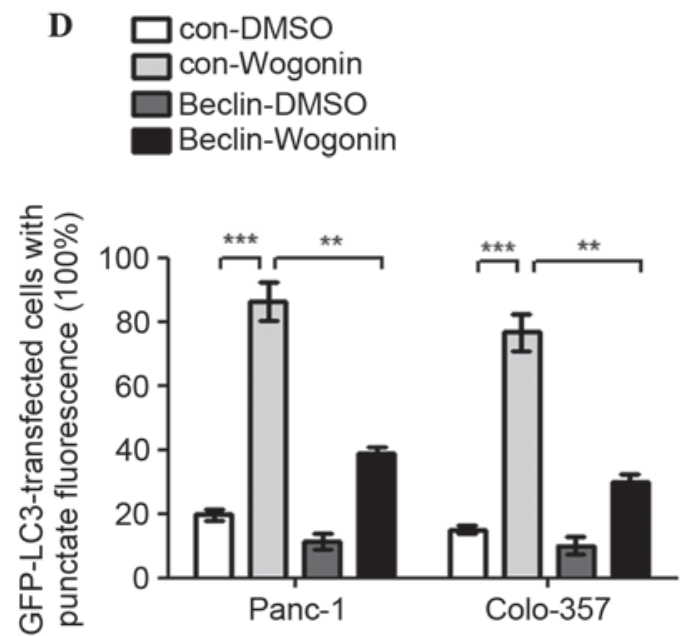

Figure 2. Wogonin activates the Beclin-1/PI3K signaling pathway. (A and B) HPCCs were treated with $40 \mu \mathrm{m}$ wogonin or $0.1 \%$ DMSO for $12 \mathrm{~h}$. Antibodies targeting (A) Beclin-1 or (B) PI3K were added to immunoprecipitate the Beclin-1- or PI3K-containing complexes and then IB for PI3K or Beclin-1 was performed. The results revealed that Beclin-1 and PI3K were pulled down after PI3K and Beclin-1 were downregulated, respectively, and wogonin could upregulate Beclin-1 and PI3K, which indicated that Beclin-1 and PI3K were bound to one another, and wogonin could promote integration to form the Beclin-1/PI3K complex. (C) HPCCs were transfected with control siRNA or siRNA-Beclin-1; at $36 \mathrm{~h}$ post-transfection, HPCCs were treated with $0.1 \%$ DMSO or $40 \mu \mathrm{M}$ wogonin for $24 \mathrm{~h}$ and then immunoblotted for LC3 and Beclin-1, revealing that LC3 expression was decreased following Beclin-1 knowckdown. (D) HPCCs expressing LC3-GFP were transfected with control siRNA or siRNA-Beclin-1. At $36 \mathrm{~h}$ post-transfection, HPCCs were treated with $0.1 \%$ DMSO or $40 \mu \mathrm{M}$ wogonin for $24 \mathrm{~h}$, and the LC3-GFP puncta-positive cells were counted ( $\geq 5$ puncta was considered positive). After Beclin-1 was knocked down, the number of positive cells was significantly decreased, but not reduced to zero, which indicated that the Beclin-1/PI3K complex was involved in, but not solely responsible for, wogonin-induced autophagy. Data are presented as the mean \pm standard deviation from triplicated experiments. ${ }^{* * *} \mathrm{P}<0.01 ;{ }^{* * * *} \mathrm{P}<0.005$. HPCC, human pancreatic cancer cell; CQ, chloroquine, LC3, microtubule-associated protein 1A/1B-light chain 3; GFP, green fluorescent protein; TEM, transmission electron microscopy; DMSO, dimethyl sulfoxide; siRNA, small interfering RNA; PI3K, phosphatidylinositol 3-kinase; GAPDH, glyceraldehyde 3-phosphate dehydrogenase; Con, control; IgG, immunoglobulin G; IB, immunoblotting; IP, immunoprecipitation.

performed on a JEOL 1230 TEM (JEOL, Tokyo, Japan) at an accelerating voltage of $80 \mathrm{kV}$. Images were acquired with an AMT Advantage Plus $2 \mathrm{~K}$ x $2 \mathrm{~K}$ digital camera connected to the TEM (18).

Small interfering RNA (siRNA) transfection experiment. Control siRNA and siRNA specifically targeted to Beclin-1 (GAGAUCUUAGAGCAAAUGATT) were purchased from RiboBio Co., Ltd. (Guangzhou, China). Cells were plated in 6-well plates and grown to $\sim 80 \%$ confluence before transient transfections with siRNAs (100 pmol per well) were performed using Lipofectamine ${ }^{\circledR} 2000$ (Invitrogen; Thermo Fisher Scientific), according to the manufacturer's instructions. After a $36-\mathrm{h}$ transfection, $0.1 \%$ DMSO or $40 \mu \mathrm{M}$ wogonin were added for an additional $24 \mathrm{~h}$ prior to collection of the cells for western blotting.

Apoptosis detection. The cells were pretreated with sterile water or $10 \mathrm{mM} \mathrm{NAC}$ for $2 \mathrm{~h}$ and then treated with $0.1 \%$ DMSO or $40 \mu \mathrm{M}$ wogonin for $24 \mathrm{~h}$, prior to collection and washing three times in PBS. The cells were then resuspended in $100 \mu \mathrm{l}$ $1 \mathrm{X}$ binding buffer $(10 \mathrm{mM}$ HEPES/NaOH $\mathrm{pH} 7.4,140 \mathrm{mM}$ $\mathrm{NaCl}$ and $2.5 \mathrm{mM} \mathrm{CaCl}_{2}$ ), and $5 \mu \mathrm{l}$ of Annexin V-fluorescein isothiocyanate (FITC) (Beyotime Institute of Biotechnology) and $5 \mu 1$ of propidium iodide (PI; Beyotime Institute of Biotechnology) were added into the suspension, which was gently vortexed and incubated at room temperature for $15 \mathrm{~min}$ in the dark. The samples were assessed using a FACSCalibur flow cytometer (BD Biosciences, Franklin Lakes, NJ, USA), and the data were analyzed by CellQuest Pro software (version 3.3; BD Biosciences) to determine the ratios of apoptotic cells.

Statistical analysis. Data are presented as the mean \pm standard deviation from triplicated experiments and SPSS 19.0 software (IBM SPSS, Armonk, NY, USA) was used to analyze the data. Two-way analysis of variance was used to analyze the differences between the groups. $\mathrm{P}<0.05$ was considered to indicate a statistically significant result. 
A

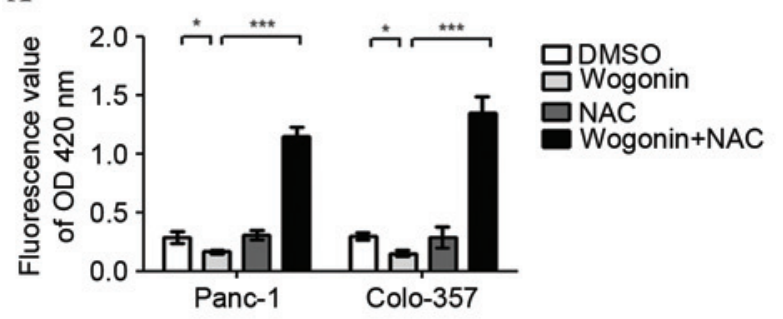

C
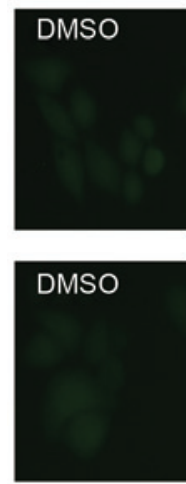
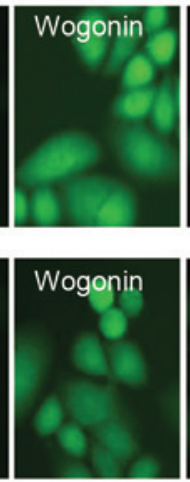
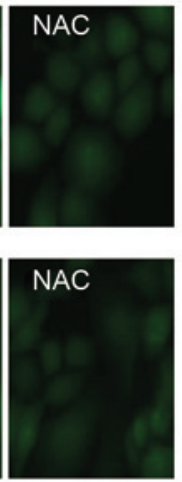

B

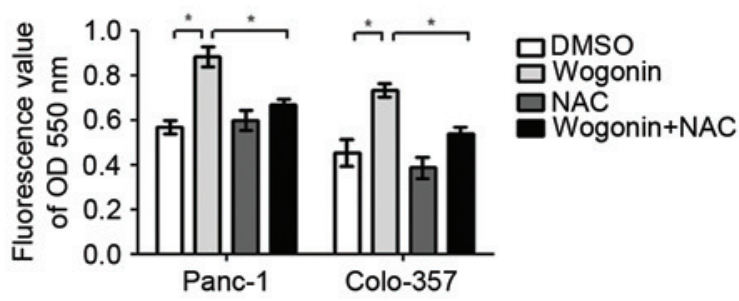

D

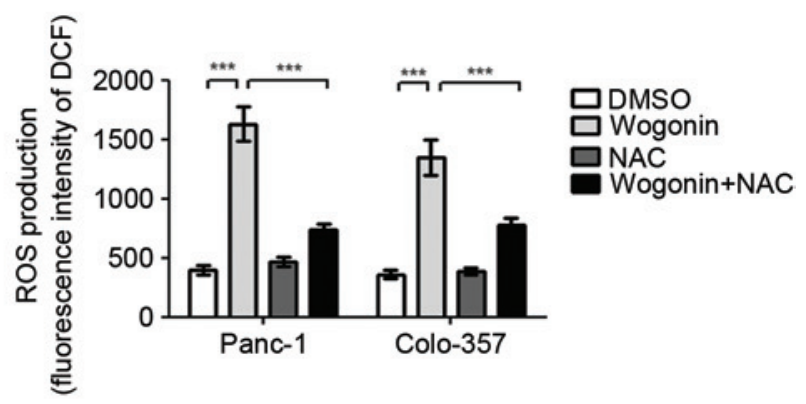

Figure 3. Wogonin may promote ROS generation in HPCCs. HPCCs were pretreated with sterile water or NAC for $2 \mathrm{~h}$ and then treated with $0.1 \%$ DMSO or $40 \mu \mathrm{M}$ wogonin for $24 \mathrm{~h}$. (A) The levels of GSH were determined using an ELISA kit at 420 nm. Wogonin reduced the intracellular levels of GSH, and wogonin combined with NAC could significantly increase the intracellular levels of GSH. (B) The levels of ${ }_{2}^{-}$were determined using an ELISA kit at $550 \mathrm{~nm}$. The intracellular levels of $\mathrm{O}_{2}^{-}$were promoted by wogonin treatment and significantly inhibited by wogonin and NAC co-treatment. The levels of ROS were evaluated by DCFH-DA using (C) a fluorescence microscope or (D) a fluorescence microplate reader. ROS generation was promoted by wogonin treatment alone, but was inhibited by wogonin and NAC co-treatment. These results indicate that wogonin promotes ROS generation in HPCCs, and the antioxidant NAC can attenuate the effect of wogonin on ROS generation. Data are presented as the mean \pm standard deviation from triplicated experiments. ${ }^{*}<0.05$; ${ }^{* * *} \mathrm{P}<0.005$. HPCC, human pancreatic cancer cell; GSH, glutathione; DMSO, dimethyl sulfoxide; ROS, reactive oxygen species; NAC, N-acetyl-cysteine; DCFH-DA, 2,7-dichlorofluorescein diacetate; OD, optical density; DCF, 2,7-dichlorofluorescein.

\section{Results}

Wogonin induces autophagy in HPCC. During autophagy, LC3-I is converted to LC3-II by lipidation through a ubiquitin-like system which involves Atg3 and Atg7; therefore, LC3 is associated with autophagic vesicles, and the presence of LC3 in autophagosomes and the presence LC3-II are the indicators of autophagy (16). In HPCCs, wogonin was observed to increase the expression levels of LC3-II; the quantified expression levels (wogonin/DMSO treatment) were 4.33 in Panc-1 cells and 1.58 in Colo-357 cells. In the rapamycin positive control group, the corresponding levels were 2.17 (Panc-1) and 3.49 (Colo-357). When wogonin was combined with CQ, an autophagy inhibitor that blocks the fusion of autophagosomes and lysosomes, an accumulation of LC3-II was observed [wogonin+CQ/DMSO levels: 4.61 (Panc-1) and 3.75 (Colo-357)] (Fig. 1A). These results indicated that wogonin could significantly increase the expression levels of LC3-II protein as compared with DMSO $(\mathrm{P}=0.038)$. The effect of wogonin on LC3-II activation was not significantly different compared with the positive control rapamycin $(\mathrm{P}=0.105)$. Furthermore, accumulation of LC3-II was observed when the autophagy inhibitor CQ combined with wogonin was used, which suggests that wogonin could activate autophagic flux at prophase.

Wogonin-induced autophagy was revealed to be time- and dose-dependent (Fig. 1B and C) with an optimal time of $24 \mathrm{~h}$ and an optimal dose of $40 \mu \mathrm{M}$. Notably, when cells were treated with $40 \mu \mathrm{M}$ wogonin for $36 \mathrm{~h}$, the LC3-II protein expression levels were decreased.

AO staining demonstrated that wogonin markedly enhanced the expression of autolysosomes, and the red fluorescence intensity was greater than for DMSO (Panc-1, $\mathrm{P}=0.003$; Colo-357, $\mathrm{P}=0.007$; Fig. 1D). Wogonin also significantly increased the quantity of LC3-GFP puncta (Panc-1, $\mathrm{P}=0.001$; Colo-357, P=0.002) (Fig. 1E). The TEM results demonstrated that wogonin significantly increased the expression of autophagosomes (Fig. 1F) and, therefore, may significantly induce autophagy in HPCC.

Wogonin induces autophagy through the Beclin-1/PI3K signaling pathway. A co-immunoprecipitation pull-down assay was used to investigate the mechanisms underlying the effects of wogonin in HPCCs. Immunoprecipitation of Beclin-1 was able to pull down PI3K, and immunoprecipitation of PI3K was able to pull down Beclin-1, indicating that Beclin-1 and PI3K are bound to each other in HPCC (Fig. 2A and B).

Beclin-1 and PI3K expression may be induced by wogonin; when Beclin-1 was knocked down with a specific siRNA, the LC3-II conversion was lower as compared with the control, although it was not absent (Fig. 2C). Similarly to wogonin treatment, the number of cells with positive GFP-LC3 puncta when Beclin-1 was knocked down was significantly decreased compared with cells in which Beclin-1 was not knocked down 
A

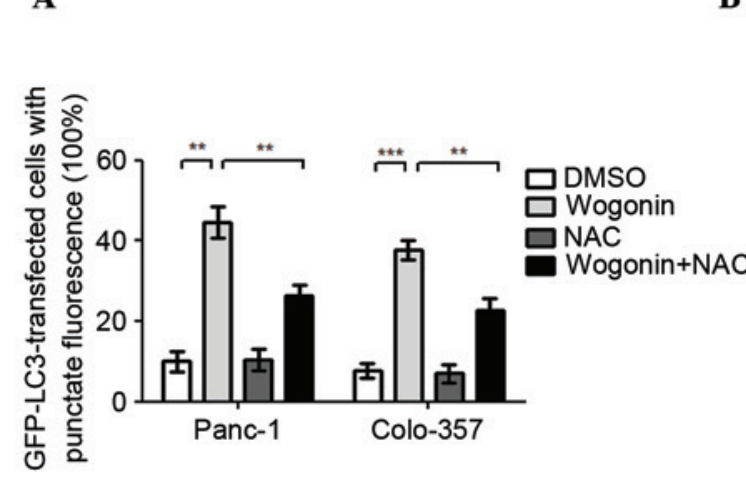

B

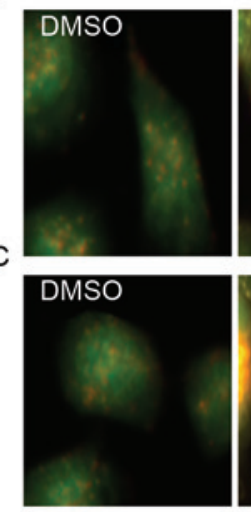

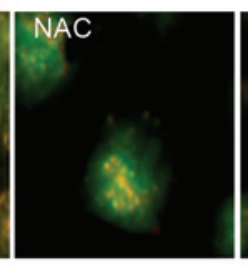
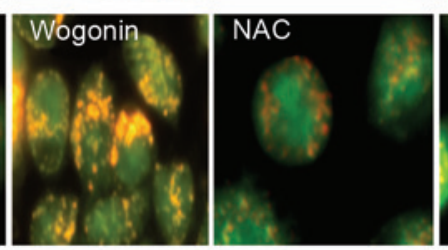
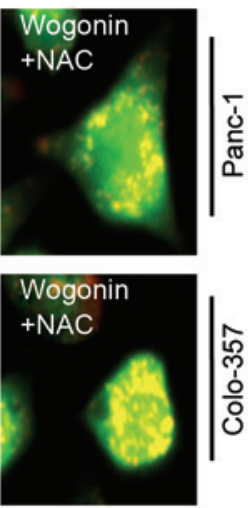

$\mathbf{C}$

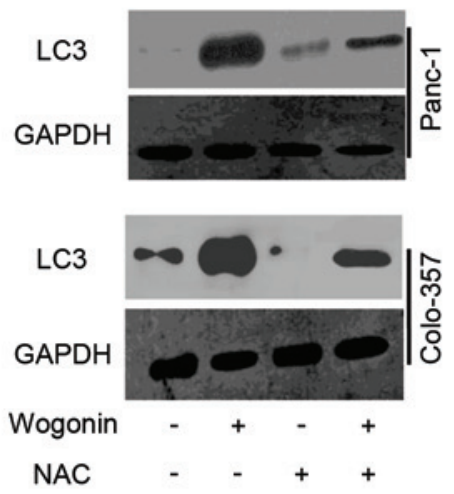

D

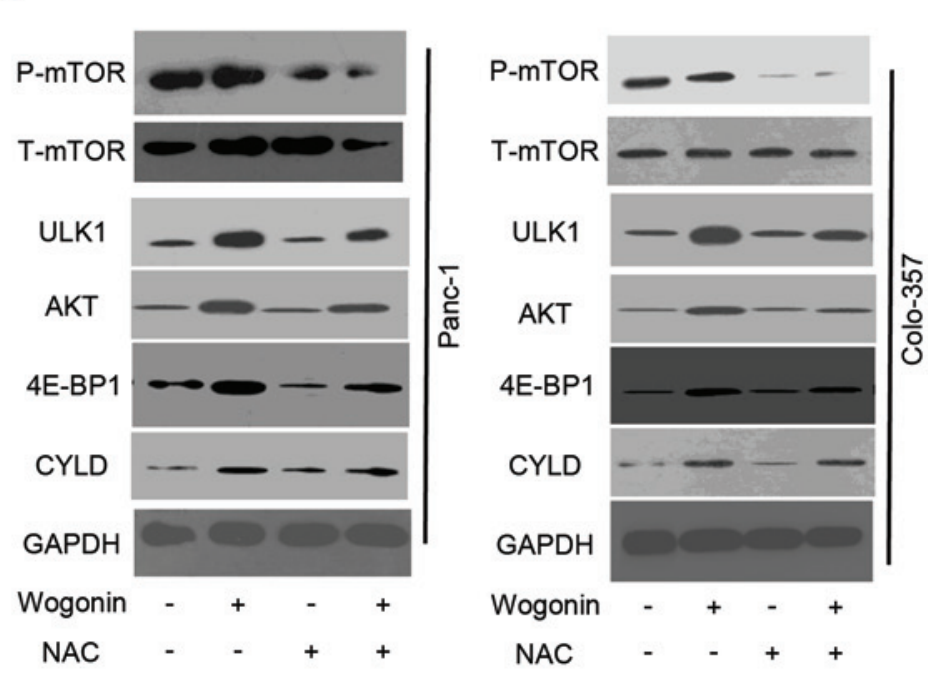

Figure 4. Wogonin induces autophagy through the ROS signaling pathway in HPCC. HPCC were pretreated with sterile water or NAC for 2 h, then treated with $0.1 \%$ DMSO or $40 \mu \mathrm{M}$ wogonin for $24 \mathrm{~h}$. (A) LC3-GFP puncta-positive cells were counted ( $\geq 5$ puncta was considered positive) and the results indicated that wogonin could increase the number of LC3-GFP puncta-positive cells, whereas co-treatment with NAC and wogonin attenuated this increase. Data are presented as the mean \pm standard deviation from triplicated experiments. ${ }^{* *} \mathrm{P}<0.01 ;{ }^{* * *} \mathrm{P}<0.005$. (B) AO staining for autolysosomes indicated that autolysosomes were activated by wogonin treatment, but inhibited by NAC and wogonin co-treatment. (C) Immunoblotting for LC3 revealed that the expression levels of LC3 were reduced by NAC and wogonin co-treatment compared with wogonin single treatment. (D) The levels of p-mTOR, t-mTOR, ULK1, AKT, 4E-BP1 and CYLD were evaluated by immunoblot. Wogonin could downregulate the expression of mTOR, and upregulate the expression levels of ULK1, AKT, 4E-BP1 and CYLD; these effects were significantly inhibited by NAC and wogonin co-treatment. AO, acridine orange; HPCC, human pancreatic cancer cell; DMSO, dimethyl sulfoxide; ROS, reactive oxygen species; NAC, N-acetyl-cysteine; LC3, microtubule-associated protein 1A/1B-light chain 3; GFP, green fluorescent protein; GAPDH, glyceraldehyde 3-phosphate dehydrogenase; mTOR, mammalian target of rapamycin; P-, phosphorylated; T-, total; ULK1, Unc-51 like autophagy activating kinase 1; AKT, protein kinase B; 4E-BP1, 4E-binding protein-1; CYLD, cylindromatosis.

(Panc-1, P=0.009; Colo-357, P=0.008; Fig. 2D). The results suggest that wogonin may induce autophagy by modulating the Beclin-1/PI3K signaling pathway.

Wogonin may promote ROS generation in HPCC. ROS was observed to mediate the survival and proliferation of cancer cells; therefore, the current study investigated the variations in ROS levels induced by wogonin in HPCCs. The results demonstrated that wogonin decreases the levels of glutathione (GSH; Fig. 3A), increases the levels of superoxide $\left(\mathrm{O}_{2}{ }^{-}\right)$and ROS (Fig. 3B-D). In addition, when the cells were co-treated with $10 \mathrm{mM}$ of the antioxidant NAC, the effect of wogonin on GSH inhibition was reversed, and the level of GSH was significantly increased (Panc-1, $\mathrm{P}=0.003$; Colo-357, $\mathrm{P}=0.001$ ). Similarly, the effects of wogonin on $\mathrm{O}_{2}{ }^{-}$and ROS generation promotion were reversed; the levels of $\mathrm{O}_{2}{ }^{-}$(Panc-1, $\mathrm{P}=0.027$;
Colo-357, $\mathrm{P}=0.031$ ) and ROS generation (Panc-1, $\mathrm{P}<0.001$; Colo-357, $\mathrm{P}<0.001$ ) were significantly decreased (Fig. 3A-D). Therefore, the results indicate that wogonin promotes ROS generation in HPCC, and the antioxidant NAC can attenuate the activation of ROS generation by wogonin.

Wogonin induces autophagy through the ROS signaling pathway in HPCC. As an antioxidant, NAC is considered as inhibitor of ROS. In order to negate the role of ROS in wogonin-induced-autophagy, HPCCs were co-treated with $10 \mathrm{mM}$ NAC and $40 \mu \mathrm{M}$ wogonin. The results demonstrated that the quantity of LC3-GFP puncta in cells co-treated with NAC and wogonin was lower, as compared with the cells treated with wogonin alone (Panc-1, $\mathrm{P}=0.007$; Colo-357, $\mathrm{P}=0.006$; Fig. 4A). AO staining also indicated that when HPCCs were co-treated with NAC and wogonin, wogonin-induced 

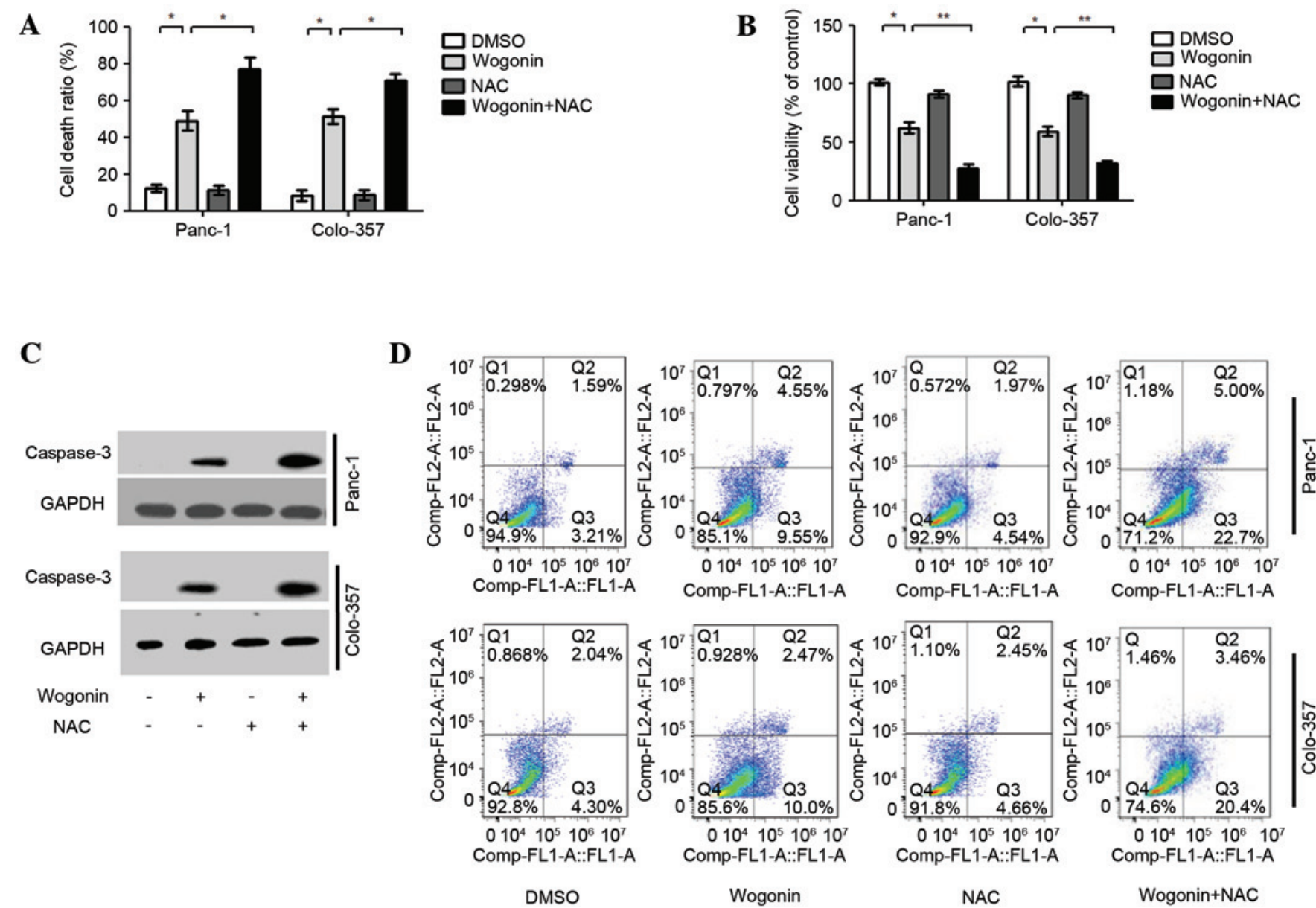

Figure 5. NAC enhances wogonin-induced apoptotic cell death. HPCCs were pre-treated with sterile water or NAC for $2 \mathrm{~h}$, then treated with $0.1 \%$ DMSO or $40 \mu \mathrm{M}$ wogonin for $24 \mathrm{~h}$. (A) Analysis of the cell death ratio by trypan blue revealed that wogonin could enhance the cell death ratio, and this effect could be further promoted by co-treatment with NAC. (B) Cell viability was determined by CCK8; cell viability was reduced by wogonin and was further inhibited by co-treatment with NAC. (C) Caspase-3 levels were assessed by immunoblotting, revaling that the expression level of caspase-3 could be upregulated by wogonin, and was further promoted by co-treatment with NAC. (D) Flow cytometry revealed that wogonin could increase the cell apoptosis ratio, and the increased effect of co-treatment with NAC was significant. Data are presented as the mean \pm standard deviation from triplicated experiments. "P<0.05; ${ }^{* *} \mathrm{P}<0.01$. HPCC, human pancreatic cancer cell; DMSO, dimethyl sulfoxide; NAC, N-acetyl-cysteine; GAPDH, glyceraldehyde 3-phosphate dehydrogenase; CCK-8, cell counting kit-8.

autophagy was inhibited by NAC (Panc-1, P<0.001; Colo-357, $\mathrm{P}<0.001$; Fig. 4B).

Similarly, LC3 expression was inhibited by NAC, as demonstrated when HPCCs were co-treated with NAC and wogonin (Fig. 4C). The results suggest that ROS may serve as an activator of wogonin-induced autophagy. The antioxidant NAC can inhibit the effect of ROS on autophagy activation. Further investigations are required to determine whether ROS are involved in additional pro-autophagy pathways in wogonin-induced autophagy.

Additional pro-autophagy pathways were detected to confirm whether they are involved in ROS-mediated autophagy following wogonin treatment. Previous reports (19-21) demonstrated that mTOR, ULK1, AKT, 4E-BP1 and CYLD participate in the mediation of autophagy. The present results revealed that wogonin could downregulate the expression of mTOR, and upregulate the expression of ULK1, AKT, 4E-BP1 and CYLD in HPCCs. When HPCCs were co-treated with wogonin and NAC, the effects of wogonin on the inhibition of mTOR, and the activation of ULK1, AKT, 4E-BP1 and CYLD were significantly attenuated. As the aforementioned results suggested that NAC could inhibit the generation of ROS in HPCCs, it is possible that mTOR, ULK1, AKT, 4E-BP1 and CYLD participate in wogonin-induced autophagy, acting as upstream signals of autophagy. In addition, these pro-autophagy molecules were mediated by ROS, acting as the downstream signals of ROS. Therefore, the pro-autophagy molecules mTOR, ULK1, AKT, 4E-BP1 and CYLD are involved in the ROS-mediated autophagy following wogonin treatment.

NAC may enhance wogonin-induced apoptotic cell death. To investigate the role of ROS-mediated autophagy in wogonin-induced-apoptotic cell death, HPCCs were treated with wogonin or co-treated with NAC and wogonin. The results demonstrated that co-treatment with NAC and wogonin was able to significantly enhance the wogonin-induced cell death ratio (Panc-1, $\mathrm{P}=0.024$; Colo-357, $\mathrm{P}=0.037$; Fig. 5A), and the ratio of cell viability was lower than in HPCCs treated with wogonin alone (Panc-1, $\mathrm{P}=0.009$; Colo-357, P=0.008; Fig. 5B). Furthermore, when HPCCs were co-treated with NAC and wogonin, the apoptotic signaling molecule caspase-3 was activated (Fig. 5C). The results of Annexin V-FITC and PI staining 
revealed that NAC could enhance the ratio of apoptotic cells induced by wogonin (Panc-1, $\mathrm{P}=0.022$; Colo-357, $\mathrm{P}=0.039$ ). Furthermore, when HPCCs were co-treated with NAC and wogonin, the apoptosis ratio of HPCCs was higher than with wogonin treatment alone (Panc-1, $\mathrm{P}=0.034$; Colo-357, $\mathrm{P}=0.026$; Fig. 5D). The results suggest that the suppression of ROS-mediated autophagy may enhance the antitumor potential of wogonin by increasing apoptosis in HPCCs.

\section{Discussion}

To the best of our knowledge, the present study is the first to demonstrate that wogonin-induced autophagy is regulated by the Beclin-1/PI3K and ROS signaling pathways. Wogonin is able to activate Beclin-1 and PI3K and promote ROS generation, inducing ROS-mediated autophagy, through activating ULK1, AKT, 4E-BP1 and CYLD, and inhibiting the mTOR signaling pathway (Fig. 6). The antitumor mechanisms underlying the role of wogonin in HPCC have been demonstrated to be an increase in apoptosis via the $\mathrm{NF}-\kappa \mathrm{B}$, signal transducer and activator of transcription 3 and PI3K/AKT signaling pathways, and induction of cell cycle arrest through modulation of the AKT/ glycogen synthase kinase $3 \beta / \beta$-catenin signaling pathways (12,22-24). Additionally, wogonin has also exhibited anti-inflammatory effects; the mechanisms underlying the anti-inflammatory effects were demonstrated to be through the regulation of the $\mathrm{NF}-\kappa \mathrm{B}$ signaling pathway that is mediated by Toll-like receptor $4 /$ myeloid differentiation primary response gene $88 /$ transforming growth factor- $\beta$-activated kinase 1 (25-27). The effects of wogonin are not isolated: The anti-inflammatory function promotes apoptosis and cell cycle inhibition, although the underlying mechanisms of these three functions require further study.

Autophagy contributes to the survival of cells under various stresses, including starvation, drug stimulation and radiation, and is therefore a protective mechanism (16). The majority of chemotherapy drugs (including sorafenib, gemcitabine and paclitaxel) can induce autophagy in cancer cells, and autophagy acts a protective mechanism for cell. Drug-induced autophagy can make cells resistant to the therapeutic effect of the drug (28-30). Therefore, it is necessary to detect whether chemotherapy drugs can induce autophagy in cancer cells, and to investigate the mechanism of chemotherapy drug-induced autophagy in order to design more appropriate treatments for patients.

In mammalian cells, PI3K can bind to Beclin-1 through special ECD and CCD domains to form the Beclin-1/PI3K core complex, and then participate in the mediation of phosphorylation and ubiquitination, eventually inducing autophagy (31). In the present study it was shown that Beclin-1 interacts with PI3K to form the Beclin-1/PI3K complex, and that wogonin promoted the interaction of Beclin-1 and PI3K, inducing autophagy in HPCCs. These results indicate that Beclin-1/PI3K is important in wogonin-induced autophagy.

ROS can aggravate cell injury by oxidative stress. As the cell reacts to the injury, autophagy is activated to promote cell survival (32). With the promotion of the ROS-enhanced oxidative stress response, induced ROS can activate the transcription factors P53, NFE2-related factor 2 and hypoxia inducible factor 1, followed by the activation of LC3, P62 and NIP3-like

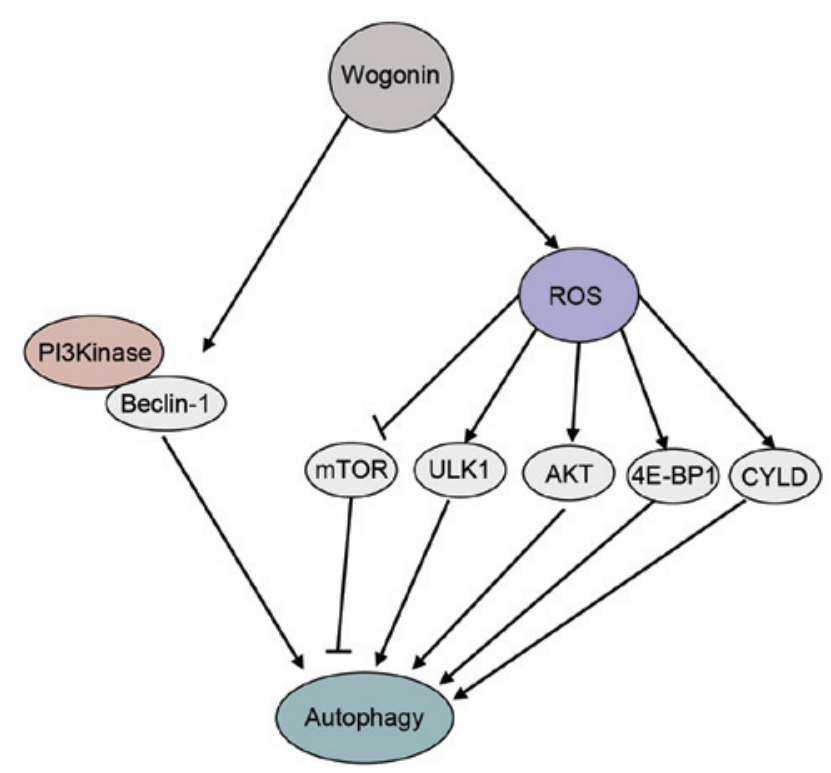

Figure 6. The signaling pathways involved in wogonin-induced autophagy. Wogonin activates Beclin-1 and PI3K. Wogonin also promotes ROS generation, inducing ROS-mediated autophagy by activating the ULK1, AKT, 4E-BP1 and CYLD signaling pathways, and inhibiting the mTOR signaling pathway. GAPDH, glyceraldehyde 3-phosphate dehydrogenase; mTOR, mammalian target of rapamycin; P-, phosphorylated; T-, total; ULK1, Unc-51 like autophagy activating kinase 1; AKT, protein kinase B; 4E-BP1, 4E-binding protein-1; PI3K, phosphatidylinositol-3-kinase; ROS, reactive oxygen species; CYLD, cylindromatosis.

protein X. Finally, the corresponding protein products can increase autophagic flux in the cytoplasm (32-35). In the present study, an increased level of ROS generation was observed in cells undergoing wogonin-induced autophagy, with upregulated $\mathrm{O}_{2}{ }^{-}$and downregulated GSH levels. Furthermore, the antioxidant NAC could block the activation of ROS generation and inhibit autophagic flux following wogonin treatment in HPCCs. These results indicate that ROS may serve as an activator in wogonin-induced autophagy in HPCCs. As previously reported, wogonin is an inhibitor of the mTOR pathway (16), and ULK1 may induce autophagy through regulation of the Beclin-1/VPS34 signaling pathway (19). Chow et al (14) reported that wogonin is able to induce autophagy and apoptosis by regulating the AKT signaling pathway. Lee et al (20) indicated that wogonin actives 4E-BP1, which is upstream of autophagy (21). The importance of CYLD in triggering autophagy has also been demonstrated (36). In the present study, wogonin was demonstrated to act as an inhibitor of mTOR and an activator of ULK1, AKT, 4E-BP1 and CYLD in HPCCs. In addition, the antioxidant NAC could attenuate the effect of wogonin on mTOR, ULK1, AKT, 4E-BP1 and CYLD, and inhibit wogonin-induced autophagy. These results indicate that the pro-autophagy molecules mTOR, ULK1, AKT, 4E-BP1 and CYLD are involved in the ROS-mediated autophagy following wogonin treatment in HPCCs. As ROS are involved in wogonin-induced autophagy, leading to inhibition of the cytotoxicity of wogonin for HPCCs, our results indicate that co-treatment with NAC and wogonin may be able to enhance wogonin-induced cell death through enhancement of apoptosis due to the ROS inhibition followed by autophagy inhibition. Thus, NAC may be a potential co-treatment with 
wogonin in HPCC, contributing to the antitumor effect of wogonin.

In summary, the findings of the present study indicate that wogonin can induce autophagy through activation of the Beclin-1/PI3K complex and triggering the ROS-mediated autophagy pathway. The pro-autophagy molecules mTOR, ULK1, AKT, 4E-BP1 and CYLD are involved in the ROS-mediated autophagy following wogonin treatment. Furthermore, the antioxidant NAC can inhibit wogonin-induced autophagy through inhibition of ROS generation, and enhance the ratio of wogonin-induced apoptotic cell death. In the future, wogonin combined with NAC may be a novel combination therapy for clinical pancreatic cancer therapy trials.

\section{References}

1. Siegel R, Naishadham D and Jemal A: Cancer statistics 2013. CA Cancer J Clin 63: 11-30, 2013.

2. Diener MK, Combs SE and Büchler MW: Chemoradiotherapy for locally advanced pancreatic cancer. Lancet Oncol 14: 269-270, 2013.

3. Akimoto M, Iizuka M, Kanematsu R, Yoshida M and Takenaga K: Anticancer effect of ginger extract against pancreatic cancer cells mainly through reactive oxygen species-mediated autotic cell death. PLoS One 10: e0126605, 2015.

4. Lewis CS, Voelkel-Johnson C and Smith CD: Suppression of c-Myc and RRM2 expression in pancreatic cancer cells by the sphingosine kinase-2 inhibitor ABC294640. Oncotarget 2016. [Epub ahead of print]

5. Mandhare A, Biradar S and Gurule A: Azaepothilone B and its derivatives: A patent review. Expert Opin Ther Pat 26: 891-905, 2016.

6. Pourmorteza M, Rahman ZU and Young M: Evofosfamide, a new horizon in the treatment of pancreatic cancer. Anticancer Drugs 27: 723-725, 2016.

7. Polier G, Ding J, Konkimalla BV, Eick D, Ribeiro N, Köhler R, Giaisi M, Efferth T, Desaubry L, Krammer PH and Li-Weber M: Wogonin and related natural flavones are inhibitors of CDK9 that induce apoptosis in cancer cells by transcriptional suppression of Mcl-1. Cell Death Dis 2: e182, 2011.

8. Polier G, Giaisi M, Köhler R, Müller WW, Lutz C, Buss EC, Krammer PH and Li-Weber M: Targeting CDK9 by wogonin and related natural flavones potentiates the anti-cancer efficacy of the Bcl-2 family inhibitor ABT-263. Int J Cancer 136: 688-698, 2015.

9. Baumann S, Fas SC, Giaisi M, Müller WW, Merling A, Gülow K, Edler L, Krammer PH and Li-Weber M: Wogonin preferentially kills malignant lymphocytes and suppresses T-cell tumor growth by inducing PLCgammal-and $\mathrm{Ca}^{2+}$-dependent apoptosis. Blood 111: 2354-2363, 2008.

10. Yang H, Hui H, Wang Q, Li H, Zhao K, Zhou Y, Zhu Y, Wang X, You Q, Guo Q and Lu N: Wogonin induces cell cycle arrest and erythroid differentiation in imatinib-resistant K562 cells and primary CML cells. Oncotarget 5: 8188-8201, 2014.

11. Takahashi H, Chen MC, Pham H, Angst E, King JC, Park J, Brovman EY, Ishiguro H, Harris DM, Reber HA, et al: Baicalein, a component of Scutellaria baicalensis, induces apoptosis by Mcl-1 down-regulation in human pancreatic cancer cells. Biochim Biophys Acta 1813: 1465-1474, 2011.

12. Kallifatidis G, Rausch V, Baumann B, Apel A, Beckermann BM, Groth A, Mattern J,LiZ, Kolb A, Moldenhauer G, et al: Sulforaphane targets pancreatic tumour-initiating cells by NF-kappaB-induced antiapoptotic signalling. Gut 58: 949-963, 2009.

13. Chen N and Karantza V: Autophagy as a therapeutic target in cancer. Cancer Biol Ther 11: 157-168, 2011.

14. Chow SE, Chen YW, Liang CA, Huang YK and Wang JS: Wogonin induces cross-regulation between autophagy and apoptosis via a variety of Akt pathway in human nasopharyngeal carcinoma cells. J Cell Biochem 113: 3476-3485, 2012.

15. Zhu Y and Wang J: Wogonin increases $\beta$-amyloid clearance and inhibits tau phosphorylation via inhibition of mammalian target of rapamycin: Potential drug to treat Alzheimer's disease. Neurol Sci 36: 1181-1188, 2015.

16. Klionsky DJ, Abdelmohsen K, Abe A, Abedin MJ, Abeliovich H, Acevedo Arozena A, Adachi H, Adams CM, Adams PD, Adeli K, et al: Guidelines for the use and interpretation of assays for monitoring autophagy (3rd edition). Autophagy 12: 1-222, 2016.
17. Steinbrenner J, Eldridge M, Tomé DF and Beynon JL: A simple and fast protocol for the protein complex immunoprecipitation (Co-IP) of effector: Host protein complexes. Methods Mol Biol 1127: 195-211, 2014.

18. Mikhaylova O, Stratton Y, Hall D, Kellner E, Ehmer B, Drew AF, Gallo CA, Plas DR, Biesiada J, Meller J and Czyzyk-Krzeska MF: VHL-regulated MiR-204 suppresses tumor growth through inhibition of LC3B-mediated autophagy in renal clear cell carcinoma. Cancer Cell 21: 532-546, 2012.

19. Russell RC, Tian Y, Yuan H, Park HW, Chang YY, Kim J, Kim H, Neufeld TP, Dillin A and Guan KL: ULK1 induces autophagy by phosphorylating Beclin-1 and activating VPS34 lipid kinase. Nat Cell Biol 15: 741-750, 2013.

20. Lee DH, Lee TH, Jung CH and Kim YH: Wogonin induces apoptosis by activating the AMPK and $\mathrm{p} 53$ signaling pathways in human glioblastoma cells. Cell Signal 24: 2216-25, 2012.

21. Thomas HE, Mercer CA, Carnevalli LS, Park J, Andersen JB, Conner EA, Tanaka K, Matsutani T, Iwanami A, Aronow BJ, et al: mTOR inhibitors synergize on regression, reversal of gene expression, and autophagy in hepatocellular carcinoma. Sci Transl Med 4: 139ra84, 2012.

22. Xiao W, Wu K, Yin M, Han S, Ding Y, Qiao A, Lu G, Deng B, Bo P and Gong W: Wogonin Inhibits Tumor-derived Regulatory Molecules by Suppressing STAT3 Signaling to Promote Tumor Immunity. J Immunother 38: 167-84, 2015.

23. Hu C, Xu M, Qin R, Chen W and Xu X: Wogonin induces apoptosis and endoplasmic reticulum stress in HL-60 leukemia cells through inhibition of the PI3K-AKT signaling pathway. Oncol Rep 33: 3146-3154, 2015.

24. Zhao L, Miao HC, Li WJ, Sun Y, Huang SL, Li ZY and Guo QL: LW-213 induces G2/M cell cycle arrest through AKT/GSK3 $\beta /$ $\beta$-catenin signaling pathway in human breast cancer cells. Mol Carcinog 55: 778-792, 2015.

25. Wang W, Xia T and Yu X: Wogonin suppresses inflammatory response and maintains intestinal barrier function via TLR4-MyD88-TAK1-mediated NF- $\kappa \mathrm{B}$ pathway in vitro. Inflamm Res 64: 423-431, 2015.

26. Lee JY and Park W: Anti-inflammatory effect of wogonin on RAW 264.7 mouse macrophages induced with polyinosinic-polycytidylic acid. Molecules 20: 6888-6900, 2015.

27. Lucas CD, Dorward DA, Sharma S, Rennie J, Felton JM, Alessandri AL, Duffin R, Schwarze J, Haslett C and Rossi AG: Wogonin induces eosinophil apoptosis and attenuates allergic airway inflammation. Am J Respir Crit Care Med 191: 626-636, 2015.

28. Prieto-Domínguez N, Ordóñez R, Fernández A, García-Palomo A, Muntané J, González-Gallego J and Mauriz JL: Modulation of autophagy by sorafenib: Effects on Treatment Response. Front Pharmacol 7: 151, 2016.

29. Kleger A, Perkhofer L and Seufferlein T: Smarter drugs emerging in pancreatic cancer therapy. Ann Oncol 25: 1260-1270, 2014.

30. Xi G, Hu X, Wu B, Jiang H, Young CY, Pang Y and Yuan H: Autophagy inhibition promotes paclitaxel-induced apoptosis in cancer cells. Cancer Lett 307: 141-148, 2011.

31. Maiuri MC, Tasdemir E, Criollo A, Morselli E, Vicencio JM, Carnuccio R and Kroemer G: Control of autophagy by oncogenes and tumor suppressor genes. Cell Death Differ 16: 87-93, 2009.

32. Scherz-Shouval R and Elazar Z: Regulation of autophagy by ROS: Physiology and pathology. Trends Biochem Sci 36: 30-38, 2011.

33. Zhang J, Kim J, Alexander A, Cai S, Tripathi DN, Dere R, Tee AR, Tait-Mulder J, Di Nardo A, Han JM, et al: A tuberous sclerosis complex signalling node at the peroxisome regulates mTORC1 and autophagyin response to ROS. Nat Cell Biol 15: 1186-1196, 2013.

34. Kim SJ, Jung HJ and Lim CJ: Reactive oxygen species-dependent down-regulation of tumor suppressor genes PTEN, USP28, DRAM, TIGAR, and CYLD under oxidative stress. Biochem Genet 51: 901-915, 2013.

35. Chen C, Liu Y and Zheng D: An agonistic monoclonal antibody against DR5 induces ROS production, sustained JNK activation and Endo G release in Jurkat leukemia cells. Cell Res 19: 984-995, 2009.

36. Bonapace L, Bornhauser BC, Schmitz M, Cario G, Ziegler U, Niggli FK, Schäfer BW, Schrappe M, Stanulla M and Bourquin JP: Induction of autophagy-dependent necroptosis is required for childhood acute lymphoblastic leukemia cells to overcome glucocorticoid resistance. J Clin Invest 120: 1310-1323, 2010. 